COMPUTATIONAL METHODS IN APPLIED MATHEMATICS, Vol.1(2001), No.2, pp.199-210

(C) Institute of Mathematics of the National Academy of Sciences of Belarus

\title{
ON THE PROBLEM OF QUADRATURE APPROXIMATION OF ONE SINGULAR INTEGRAL OPERATOR
}

\author{
J. SANIKIDZE \\ N. Muskhelishvili Institute of Computational Mathematics, Georgian Academy of Sciences \\ 8 Akuri Str., 380093 Tbilisi, Georgia \\ E-mail: jemal@nummath.acnet.ge
}

\begin{abstract}
A computational scheme for approximating a singular operator in the known Lippman-Schwinger equations is suggested. It is based on partial use of Gaussian knots. On the basis of studying the asymptotic properties of the solution and one unclassical estimation of the accuracy of the Gaussian quadrature formulas, the convergence of the scheme, with estimation of the rate of convergence, is proved. The analysis of the computational aspects of the scheme is carried out and the results of calculations of some test examples are given.
\end{abstract}

2000 Mathematics Subject Classification: 65N20; 65M25.

Keywords: singular integral, Gaussian quadrature formula, numerical scheme, Lippman-Schwinger equation, convergence, estimation.

The aforementioned singular operators are connected with the problem of dispersion in the quantum theory in impulse representation, which can be reduced to the solution of integral equations of certain form with singular kernels - the Lippman-Schwinger equations for $t$-dispersion matrices $[1,3,7,11]$. These equations can be written in the following form:

$$
T\left(x ; x_{0}\right)+\frac{2}{\pi} \int_{0}^{+\infty} \frac{K(x, y)}{y^{2}-x_{0}^{2}} T\left(y ; x_{0}\right) d y=K\left(x, x_{0}\right) \quad(x \geqslant 0),
$$

where the parameter $x_{0}$ is arbitrarily fixed in the interval $(0,+\infty)$ and the singular integral (with fixed singularity at the point $\left.x_{0}\right)$ is the principal value. $K(x, y)^{\prime} s \quad(x, y \geqslant 0)$ are the given "potential" functions, $T\left(y, x_{0}\right)$ is an unknown function, which defines in the known way the so-called phase of the nucleon-nucleon dispersion.

The question of numerical solution of equations of form (1) with the appropriate foundation (statement of solvability of the approximating equations and convergence of corresponding solutions to the desired function) is of considerable interest. However, it should be noted that in spite of this and the existence of some practically used numerical algorithms (see, e.g., [2]) applied to the equations of form (1) the problem of giving foundation for similar computational schemes has not as yet been solved. The main difficulty here is apparently posed by a certain singularity of kernels (potentials) $K(x, y)$ considered in dispersion problems. This singularity, which manifests itself mainly in the character of the 
behavior of the corresponding functions $K(x, y)$ in the neighborhood of the infinity, makes the judgement on the possibility of some effective approximation of singular operator in the equation (1)substantially difficult whatever the scheme may be. Thus, it turns out that it is difficult to substantiate even the first, though significant step, i.e., the approximation of the very initial equation. Considerations in the paper are mainly devoted to this problem. Namely, a certain process of approximation (of quadrature type) of singular integrals in (1) is suggested and an estimate of the convergence rate is given.

It was mentioned above that the structure properties of the function $K(x, y)$ influence the efficiency of the approximation of corresponding singular integral operators. The simplest but characteristic in the sense indicated is the case of Yukawa's potential (see, e.g., [1]):

$$
K(x, y)=\frac{v_{0}}{4} \ln \frac{(y+x)^{2}+\eta^{2}}{(y-x)^{2}+\eta^{2}} \quad\left(v_{0}=-\frac{50}{41.47}, \eta=.7\right),
$$

which we shall bear in mind in further considerations.

Here we shall not formulate the known results (see, e.g., [9]) concerning the solvability of the equations of form (1). As a result, assuming that for any fixed $x_{0}>0$ the initial equation has a solution, we shall prove some properties of the corresponding solution, which, in particular, concerns its behavior in the neighborhood of the infinity. The mentioned properties are mainly used for obtaining the needed estimates.Here, as already noted above, $K(x, y)$ is everywhere understood to represent the expression given by formula (2).

Considering the parameter $x_{0} \in(0,+\infty)$ arbitrarily fixed, we denote

$$
Q(T ; x) \equiv \int_{0}^{+\infty} \frac{K(x, y) T\left(y ; x_{0}\right)}{y^{2}-x_{0}^{2}} d y
$$

whereby $T=T\left(x ; x_{0}\right)$ the solution of equation $(1)$ is meant.

The proof of the properties of the function $T$ which is of interest to us is essentially based on the statements given below in Sections 1, 2. Now it is supposed (yet formally) that the values in the corresponding estimates are a priori finite.

1. The asymptotic estimates

$$
\begin{gathered}
|Q(T ; x)| \leqslant \frac{c_{0} M(T) \ln x}{x} \quad(x \rightarrow+\infty), \\
\left|\frac{d Q(T ; x)}{d x}\right| \leqslant \frac{c_{1} M(T)+c_{1}^{\prime}}{x^{2}} \quad(x \rightarrow+\infty),
\end{gathered}
$$

are right, where

$$
M(T)=\sup \left|T\left(x ; x_{0}\right)\right|+\sup _{x_{1}, x_{2} \in\left[0,2 x_{0}\right]} \frac{\left|T\left(x_{1} ; x_{0}\right)-T\left(x_{2} ; x_{0}\right)\right|}{\left|x_{1}-x_{2}\right|^{\beta}} \quad\left(o<\beta \leqslant 1, \quad x_{1} \neq x_{2}\right),
$$

and the constants $c_{0}, c_{1}, c_{1}^{\prime}$ do not depend on $x$.

Really, divide (assuming $x>4 x_{0}$ ) the integral in $Q(T ; x)$ as follows:

$$
\int_{0}^{2 x_{0}}+\int_{2 x_{0}}^{x / 2}+\int_{x / 2}^{+\infty}
$$


For the first integral we have (sinse $K(x, y)>0$ )

$$
\begin{aligned}
\left|\int_{0}^{2 x_{0}}\right| \leqslant & \frac{K\left(x, x_{0}\right)\left|T\left(x_{0} ; x_{0}\right)\right|}{2 x_{0}} \int_{0}^{2 x_{0}} \frac{d y}{y+x_{0}}+\int_{0}^{2 x_{0}} \frac{K(x, y)\left|T\left(y ; x_{0}\right)-T\left(x_{0} ; x_{0}\right)\right|}{\left|y-x_{0}\right|\left(y+x_{0}\right)} d y \\
& +\left|T\left(x_{0} ; x_{0}\right)\right| \int_{0}^{2 x_{0}} \frac{\left|K(x, y)-K\left(x, x_{0}\right)\right|}{\left|y-x_{0}\right|\left(y+x_{0}\right)} d y \\
\leqslant & \left\{a_{1} K\left(x, x_{0}\right)+a_{2} \max _{a \leqslant y \leqslant 2 x_{0}} K(x, y)+2 x_{0} a_{1} \max _{0 \leqslant y \leqslant 2 x_{0}}\left|K_{y}^{\prime}(x, y)\right|\right\} M(T) \quad\left(K_{y}^{\prime}=\partial K / \partial y\right)
\end{aligned}
$$

where $a_{1}, \quad a_{2}$ are known constants (depending on $\beta$ and $^{1} x_{0}$ ).

Having used the elementary estimates of the functions $K(x, y)$ and $K_{y}^{\prime}(x, y)$ in the given interval, we come to the conclusion that the expression in the braces is bounded for $x \geqslant 0$ and is $O(1 / x)$ for $x \rightarrow+\infty$.

The absolute value of the second integral in (4) does not exceed

$$
\int_{2 x_{0}}^{x / 2} \frac{K(x, y)\left|T\left(y ; x_{0}\right)\right|}{y^{2}\left(1-x_{0}^{2} / y^{2}\right)} d y \leqslant \frac{4}{3} M(T) \int_{2 x_{0}}^{x / 2} \frac{K(x, y)}{y^{2}} d y .
$$

Integrating by parts the right-hand side of (5):

$$
\int_{2 x_{0}}^{x / 2} \frac{K(x, y)}{y^{2}} d y=\frac{K\left(x, 2 x_{0}\right)}{2 x_{0}}-\frac{2 K(x, x / 2)}{x}+\int_{2 x_{0}}^{x / 2} \frac{K_{y}^{\prime}(x, y)}{y} d y,
$$

we see that the first two terms are bounded in the considered interval and are $O(1 / x)$ $(x \rightarrow+\infty)$. For the integral on the right-hand side of (6), taking into account that

$$
\max _{2 x_{0} \leqslant y \leqslant x / 2}\left|K_{y}^{\prime}(x, y)\right|=O\left(\frac{1}{x}\right) \quad(x \rightarrow+\infty),
$$

we come to

$$
\int_{2 x_{0}}^{x / 2} \frac{K_{y}^{\prime}(x, y)}{y} d y=O\left(\frac{\ln x}{x}\right) \quad(x \rightarrow+\infty) .
$$

Concerning the integral in the interval $[x / 2,+\infty)$, via evident estimate

$$
K(x, y)=O(\ln y) \quad\left(y \geqslant \frac{x}{2} ; x \rightarrow+\infty\right),
$$

we can write

$$
\begin{gathered}
\left|\int_{x / 2}^{+\infty} \frac{K(x, y)}{y^{2}-x_{0}^{2}} T\left(y ; x_{0}\right) d y\right| \leqslant a_{3} \sup _{y \geqslant 0}\left|T\left(y ; x_{0}\right)\right| \int_{x / 2}^{+\infty} \frac{\ln y}{y^{2}} d y \leqslant a_{4} M(T) \frac{\ln x}{x} \\
\left(x \rightarrow+\infty ; \quad a_{3}, a_{4}=\text { const }\right) .
\end{gathered}
$$

\footnotetext{
${ }^{1}$ Though, as a matter of fact, only the constant $a_{2}$ depends on $\beta$.
} 
Putting together the estimates obtained we come to (3).

Now (since $\left.K\left(x, x_{0}\right)=O(1 / x)(x \rightarrow+\infty)\right)$ from equation (1) on the basis of the estimate obtained we get

$$
\left|T\left(x ; x_{0}\right)\right| \leqslant a_{5} \frac{M(T) \ln x}{x}+O\left(\frac{1}{x}\right) \quad\left(x \rightarrow+\infty ; \quad a_{5}=\text { const }\right) .
$$

Using this estimate again in integrals on the intervals $\left[2 x_{0}, x / 2\right],[x / 2,+\infty)$, after elementary transformations we obtain a more exact estimate:

$$
\left|T\left(x ; x_{0}\right)\right| \leqslant \frac{c_{0} M(T)+c_{0}^{\prime}}{x} \quad\left(x \geqslant 4 x_{0} ; \quad c_{0}^{\prime}=\text { const }\right) .
$$

Establishment of inequality $\left(3_{1}\right)$ is also based on the estimation of the integrals obtained in the given case by similar division of the integral

$$
\int_{0}^{+\infty} \frac{K_{x}^{\prime}(x, y) T\left(y ; x_{0}\right)}{y^{2}-x_{0}^{2}} d y
$$

noting here that in our considerations the differentiation under the integral sign $Q(T ; x)$ is possible.

First of all we notice that for the integral on $\left[0,2 x_{0}\right]$, since

$$
\max \left\{\max _{0 \leqslant y \leqslant 2 x_{0}}\left|K_{x}^{\prime}(x, y)\right|, \max _{0 \leqslant y \leqslant 2 x_{0}}\left|K_{x y}^{\prime \prime}(x, y)\right|\right\}=O\left(\frac{1}{x^{2}}\right) \quad\left(x \geqslant 4 x_{0}\right),
$$

analogously to the previous case it is not difficult to get the estimate

$$
\left|\int_{0}^{2 x_{0}} \frac{K_{x}^{\prime}(x, y) T\left(y ; x_{0}\right)}{y^{2}-x_{0}^{2}} d y\right| \leqslant \frac{a_{6} M(T)}{x^{2}} \quad\left(x \geqslant 4 x_{0}\right),
$$

where the constant $a_{6}$ does not depend on $x$.

Further, using the estimate (7), we can write

$$
\left|\int_{2 x_{0}}^{x / 2} \frac{K_{x}^{\prime}(x, y) T\left(y ; x_{0}\right)}{y^{2}-x_{0}^{2}} d y\right| \leqslant\left[a_{7} M(T)+a_{8}\right] \int_{2 x_{0}}^{x / 2} \frac{\left|K_{x}^{\prime}(x, y)\right|}{y^{3}} d y \quad\left(a_{7}, a_{8}=\text { const }\right) .
$$

It can be verified directly that the function $K_{x}^{\prime}(x, y)$ for a sufficiently large $x$ and $y \leqslant x / 2$ maintains a sign. Taking this into account, on the basis of the equality

$$
\int_{2 x_{0}}^{x / 2} \frac{K_{x}^{\prime}(x, y)}{y^{3}} d y=-\frac{2 K_{x}^{\prime}(x, x / 2)}{x^{2}}+\frac{K_{x}^{\prime}\left(x, 2 x_{0}\right)}{8 x_{0}^{2}}+\frac{1}{2} \int_{2 x_{0}}^{x / 2} \frac{K_{x y}^{\prime \prime}(x, y)}{y^{2}} d y
$$

and directly obtained estimate

$$
\max _{2 x_{0} \leqslant y \leqslant x / 2}\left|K_{x y}^{\prime \prime}(x, y)\right|=O\left(\frac{1}{x^{2}}\right) \quad\left(x \geqslant 4 x_{0}\right)
$$


we come to the fact that the absolute value of the considered integral for sufficiently large $x$ does not exceed an expression of the form

$$
\frac{a_{9} M(T)+a_{10}}{x^{2}} \quad\left(a_{9}, a_{10}=\text { const }\right)
$$

An analogous estimate can be obtained also for the corresponding integral on $\left[\frac{x}{2},+\infty\right)$.

Remark 1. Using the direct estimates of the integrals

$$
\int_{2 x_{0}}^{x / 2} \frac{K_{x}^{\prime}(x, y) T\left(y ; x_{0}\right)}{y^{2}-x_{0}^{2}} d y, \quad \int_{x / 2}^{+\infty} \frac{K_{x}^{\prime}(x, y) T\left(y ; x_{0}\right)}{y^{2}-x_{0}^{2}} d y
$$

(by $\sup |T|$ ) we can come to the following (compare with (3)):

$$
\left|\frac{d}{d x} Q(T ; x)\right| \leqslant \frac{c_{0}^{\prime \prime} M(T)}{x} \quad\left(x \rightarrow+\infty ; \quad c_{0}^{\prime \prime}=\text { const }\right) .
$$

On the basis of similar considerations it is not difficult to get an estimate for the corresponding integral operator $Q(T ; x)$ for $x \leqslant x_{0}$, which together with the previous judgement shows that $Q(T ; x)$, as well as $\frac{d Q(T ; x)}{d x}$, and as a result - $\left|T\left(x ; x_{0}\right)\right|,\left|T^{\prime}\left(x ; x_{0}\right)\right|$ have an estimate $a_{11} M(T)+a_{12}$ with some constants $a_{11}, a_{12}$. Accordingly (compare with (7)) for sufficiently large $x \quad\left(x>4 x_{0}\right)$

$$
\left|T^{\prime}\left(x ; x_{0}\right)\right| \leqslant \frac{c_{1} M(T)+c_{1}^{\prime \prime}}{x^{2}} \quad\left(c_{1}, c_{1}^{\prime \prime}=\text { const }\right)
$$

2. The estimate

$$
\left|T^{\prime \prime}\left(x, x_{0}\right)\right| \leqslant c_{2} M(T)+c_{2}^{\prime},
$$

holds where constants $c_{2}, c_{2}^{\prime}$ do not depend on $x$.

This estimate can be obtained on the basis of transformations analogous to the previous ones. Moreover, it should be noted that in the given case also similarly to $\left(7_{1}\right)$ asymptotic dependence on $x \rightarrow+\infty$ can also be stated (but such estimate is not needed in further considerations).

We now present the quadrature process ${ }^{2}$, described in this paper for the considered singular integral $Q(T ; x)$. Using the change of variable $y=x_{0}(-1+1 / t)$, the indicated integral can be reduced to the form

$$
\frac{1}{4 x_{0}} \int_{-1}^{+1} \frac{\left[K(x, \bar{y}) T(\bar{y} ; x)-K\left(x, x_{0}\right) T\left(x_{0} ; x_{0}\right)\right]}{|t|-1 / 2} d t,
$$

where $\bar{y}=x_{0}(-1+1 /|t|)$. Furthermore, setting the natural number $n$ given arbitrarily, we approximate the last integral by the sum

$$
\frac{1}{4 x_{0}} \sum_{\nu=1}^{n} A_{\nu} \frac{K\left(x, \bar{y}_{\nu}\right) T\left(\bar{y}_{\nu} ; x_{0}\right)-K\left(x, x_{0}\right) T\left(x_{0} ; x_{0}\right)}{\left|t_{\nu}\right|-1 / 2}
$$

\footnotetext{
${ }^{2}$ In this case we give one of the simplest variants of similar schemes that were used by us in computational experiments.
} 
where $t_{\nu} \quad(\nu=1,2, \ldots, n)$ and $A_{\nu}\left(A_{\nu}>0 ; \nu=1,2, \ldots, n\right)$ are the Gaussian knots and coefficients, respectively.Then, using the known asymptotic representations of $t_{\nu}[10 ; \S 8.9]$, we can see that $\left|t_{\nu}\right| \neq 1 / 2$. The expression $x_{0}\left(-1+1 /\left|t_{\nu}\right|\right) \quad\left(t_{\nu} \neq 0\right)$ is denoted by $\bar{y}_{\nu}$, taking into account that for $t_{\nu}=0$ (which holds only for odd $n$ ) $\lim _{y \rightarrow+\infty} K(x, y)=0$ for arbitrarily given $x \geqslant 0$. For definiteness we mean everywhere below, that $n$ is an even number. Under the given assumptions, via the elementary properties of the numbers $t_{\nu}, A_{\nu}$, the corresponding quadrature process can be finally presented by the formula

$$
\int_{0}^{+\infty} \frac{K(x, y) T(y ; x)}{y^{2}-x_{0}^{2}} d y \approx-\frac{1}{2 x_{0}} \sum_{\nu=1}^{n / 2} A_{\nu} \frac{K\left(x, y_{\nu}\right) T\left(y_{\nu} ; x\right)-K\left(x ; x_{0}\right) T\left(x_{0} ; x_{0}\right)}{t_{\nu}-1 / 2}
$$

in which

$$
y_{\nu}=x_{0}\left(-1+1 / t_{\nu}\right)
$$

The quadrature process constructed in this way uses the knots $t_{\nu}$ and the coefficients $A_{\nu}$ of the ordinary Gaussian formula for the segment $[-1,+1]$, but differs from it by using only the knots of the interval $(0,1)$. The convergence and exactness of this quadrature process, in addition to the differential properties of the integrand function over the entire segment $[-1,+1]$, depend also on its certain local properties in the neighborhood of the midpoint of the segment.

Our further aim is to get an estimate of the error of the approximate process (8) which would be uniform on $[0,+\infty)$. We note that the estimate of the corresponding remainder term for fixed values of the parameter $x$ with indication of the convergence rate can be obtained using the known classical estimates from the theory of quadrature formulas. However, obtaining similar estimates in the same way for $x \rightarrow+\infty$ and by the estimate uniform on the whole interval $[0,+\infty)$ with statement of convergence of the process turns out to be difficult. Nevertheless, the needed estimation for the remainder of (8) becomes possible by using the indicated one auxiliary estimate of the Gauss quadrature formula with an even number of knots. ${ }^{3}$ The auxiliary estimate mentioned and its proof are given below in Section 3.

3. Let $\varphi(t)$ be such a given (integrable) function on the segment $[-1,+1]$ that the product $t \varphi(t)$ satisfies the Hölder condition on this interval with the index $\alpha(0<\alpha \leqslant 1)$. Then for the remainder $r_{n}(\varphi)$ of the Gauss quadrature

$$
\int_{-1}^{+1} \varphi(t) d t \approx \sum_{\nu=1}^{n / 2} A_{\nu}\left\{\varphi\left(t_{\nu}\right)+\varphi\left(-t_{\nu}\right)\right\}
$$

the following estimate is true:

$$
\left|r_{n}(\varphi)\right| \leqslant \frac{c_{3} L(t \varphi)}{n^{\alpha}} \ln n \quad(n>1)
$$

where $L(t \varphi)$ is the Hölder constant of the function $t \varphi(t)$, and the constant $c_{3}$ does not depend on $n$ and $\varphi$.

\footnotetext{
${ }^{3}$ We note that the corresponding estimate can be generalized for the case of odd numbers of knots.
} 
In order to prove this statement, we shall start from some considerations of paper [8] that concerns the quadrature process for singular integral with Jacobi weight. First we note that the mentioned process for $p=q=0, x=0$ and even $n$ goes over into the relation

$$
\int_{-1}^{+1} \frac{\psi(t)}{t} d t \approx \sum_{\nu=1}^{n / 2} \frac{A_{\nu}}{t_{\nu}}\left\{\psi\left(t_{\nu}\right)-\psi\left(-t_{\nu}\right)\right\}
$$

which has an algebraic order of exactness $2 n$ (see, e.g., [6]). According to one of the statements in [8], we can write

$$
\sum_{\nu=1}^{n / 2} \frac{A_{\nu}}{t_{\nu}} \leqslant c_{4} \ln n \quad\left(n>1 ; \quad c_{4}=\text { const }\right) .
$$

Using further reasoning from the same paper [8], on condition that the function $\psi(t)$ satisfies the Hölder condition on the segment $[-1,+1]$, we can write

$$
\left|\int_{-1}^{+1} \frac{\psi(t)}{t} d t-\sum_{\nu=1}^{n / 2} \frac{A_{\nu}}{t_{\nu}}\left[\psi\left(t_{\nu}\right)-\psi\left(-t_{\nu}\right)\right]\right| \leqslant \frac{c_{5} L(\psi)}{n^{\alpha}} \ln n \quad\left(n>1 ; c_{5}=\text { const }\right) .
$$

Changing in this estimation and in $(11) \psi(t)$ by $t \varphi(t)$, we come to the ordinary Gauss quadrature process (9) with estimate (10) of the remainder term.

Unlike the known classical estimations, the one given here states the convergence of the Gauss quadrature process for some classes of unbounded functions.

In order to apply the indicated result to obtain the estimation of interest to us, we must show that the function

$$
\Phi(T ; x,|t|) \equiv \frac{\left[K(x, \bar{y}) T(\bar{y} ; x)-K\left(x, x_{0}\right) T\left(x ; x_{0}\right)\right] t}{|t|-1 / 2} \quad(|t| \neq 1 / 2)
$$

(extended for $|t|=1 / 2$ as corresponding limit) satisfies the conditions, indicated in Section 3, (for $\alpha=1$ ) uniformly with respect to $x \geqslant 0$. For this let us convince ourselves that under the conditions 1)-2) the derivative $\frac{\partial \Phi}{\partial t}$ on each segment ${ }^{4}[-1,0],[0,1]$ is bounded uniformly with respect to $x \geqslant 0$. Evidently, it is sufficient to consider $t \in[0,1]$.

Fixing $\delta \in(0,1 / 2)$, first we shall show the estimate of the derivative

$$
\frac{\partial \Phi(T ; x, t)}{\partial t}
$$

for $t \in[1 / 2-\delta, 1]$. Obviously we can write

$$
\begin{gathered}
\max _{1 / 2-\delta \leqslant t \leqslant 1}\left|\frac{\partial \Phi}{\partial t}\right| \leqslant \max _{1 / 2-\delta \leqslant t \leqslant 1}\left|\frac{\partial}{\partial t}\left[K(x, y) T\left(y ; x_{0}\right)\right]\right|+\frac{1}{2} \max _{1 / 2-\delta \leqslant t \leqslant 1}\left|t \frac{\partial^{2}}{\partial t^{2}}\left[K(x, y) T\left(y ; x_{0}\right)\right]\right| \\
\left(y=x_{0}(-1+1 / t)\right) .
\end{gathered}
$$

Doing the appropriate differentiation and noting that the derivatives

$$
\frac{\partial}{\partial t} K(x, y), \quad \frac{\partial^{2}}{\partial t^{2}} K(x, y)
$$

\footnotetext{
${ }^{4}$ The existence of the derivative at the very point $t=0$ is not stated.
} 
are bounded for $1 / 2-\delta \leqslant t \leqslant 1$ uniformly with respect to $x \geqslant 0$, using the estimates of Sections 1, 2, we obtain

$$
\max _{t \in[1 / 2-\delta, 1]}\left|\frac{\partial \Phi(T ; x, t)}{\partial t}\right| \leqslant c_{6} M(T)+c_{7} \quad(x \geqslant 0),
$$

where the constants $c_{6}, c_{7}$ can be considered independent of $x$. Further, setting $t \in(0,1 / 2-$ $\delta$ ), we shall use the evidently obtained estimate

$$
\begin{aligned}
\left|\frac{\partial \Phi}{\partial t}\right| \leqslant & \frac{1}{\delta}\left(1+\frac{t}{\delta}\right)\left\{K(x, y)\left|T\left(y ; x_{0}\right)\right|+K\left(x, x_{0}\right)\left|T\left(x_{0} ; x_{0}\right)\right|\right\} \\
& +\frac{1}{\delta}\left\{\left|K_{t}^{\prime}(x, y) t T\left(y ; x_{0}\right)\right|+K(x, y) t\left|T_{t}^{\prime}\left(y ; x_{0}\right)\right|\right\} .
\end{aligned}
$$

First of all, let us note that for $0<t<1 / 2-\delta$ the product $t K(x, y)$ is bounded (and approaches zero as $t \rightarrow 0$ ) uniformly with respect to $x \geqslant 0$. Clearly, fixing $p>1$, it is easy to follow that for $x, y \rightarrow \infty$, satisfying $x \geqslant p y$, the function $K(x, y)$ is bounded and for $x<p y$ via the evident estimate

$$
K(x, y) \leqslant \ln \frac{(x+y)^{2}+\eta^{2}}{\eta^{2}}
$$

we can write

$$
K(x, y)=O(\ln y) \quad(y \rightarrow+\infty)
$$

(uniformly with respect to $x<p y$ ). This (remembering that $y=x_{0}(-1+1 / t$ )) yields our statement, due to which, via the inequality (7), it is easy to obtain (uniformly with respect to $x>0$ )the estimate for

$$
\sup _{t \in(0,1 / 2-\delta)} K(x, y)|T(y ; x)|
$$

by $M(T)$. Further, via the equalities

$$
K_{t}^{\prime}(x, y)=-x_{0} \frac{K_{y}^{\prime}(x, y)}{t^{2}}, \quad T_{t}^{\prime}\left(y ; x_{0}\right)=-x_{0} \frac{T^{\prime}\left(y ; x_{0}\right)}{t^{2}},
$$

and taking into account that the function $K_{y}^{\prime}(x, y)$ is bounded for $x, y \geqslant 0$, remembering the results of Sections 1, 2 and elementary estimate $K\left(x, x_{0}\right)\left|T\left(x ; x_{0}\right)\right|=O(1) M(T) \quad(x \geqslant 0)$, we have from $(12)$

$$
\sup _{t \in(0,1 / 2-\delta)}\left|\frac{\partial \Phi(T ; x, t)}{\partial t}\right| \leqslant c_{8} M(T)+c_{9} \quad(x \geqslant 0)
$$

(with the reservation, analogous to the above, concerning the constants $c_{8}, c_{9}$ ). Finally, putting together the obtained inequalities for the remainder term $R_{n}(T ; x)$ of the quadrature process (8) via (10), we come to the estimate

$$
\sup _{x \geqslant 0}\left|R_{n}(T ; x)\right| \leqslant\left[c_{10} M(T)+c_{11}\right] \frac{\ln n}{n} \quad(n>1)
$$

where the constants $c_{10}, \quad c_{11}$ are independent of $n$ and $x$ and can be defined effectively.

It can be shown that under the assumption

$$
\|\psi\|=\sup _{x \geqslant 0}|\psi|+\sup _{x_{1}, x_{2} \geqslant 0} \frac{\left|\psi\left(x_{1}\right)-\psi\left(x_{2}\right)\right|}{\left|x_{1}-x_{2}\right|^{\beta}} \quad\left(0<\beta<1, x_{1} \neq x_{2}\right)
$$


on the set of the functions $\psi(x)$ that in $[0,+\infty)$ satisfy the Hölder condition with index $\beta \in(0,1)$ and that have the finite limit at $x \rightarrow+\infty$, the integral operator $Q(\psi ; x)$ represents a completely continuous operator in the respective (Banach) space and on condition of the solvability of the given equation, $M(T)$ is a finite value. In such a case, as follows from the obtained inequality for $R_{n}(T ; x)$, the rate of approximation of the considered equation by the given scheme is $O\left(\frac{\ln n}{n}\right) \quad(n>1)$. The complete substantiation of the scheme needs certain additional considerations which, however, are rather bulky, and it seems reasonable to consider them separately. Here we will give (at the end of the paper)only some results of numerical experiments connected with the solution of equation (1).

We note that using similar considerations, due to the properties of the function $K(x, y)$ and its derivatives, the analogous estimate can be obtained for the expression

$$
\sup _{x_{1}, x_{2} \geqslant 0} \frac{\left|R_{n}\left(T ; x_{1}\right)-R_{n}\left(T ; x_{2}\right)\right|}{\left|x_{1}-x_{2}\right|^{\beta}}\left(x_{1} \neq x_{2}\right) .
$$

We also note that in the proof of the convergence of the process considered it is important that in the given scheme of transformations of the initial integral operator the inner point (the midpoint in this case) of the segment $[-1,+1]$ could correspond to the infinity. This essentially gives the possibility of using effectively estimate (10). In [2] the corresponding singular integral, after changing $y=\tan \pi t / 2(0 \leqslant t<1)$, on the interval $[0,+\infty)$ is approximated directly by the Gaussian quadrature formula. For such a scheme of approximation (containing the mapping of the infinity to the end of the segment $[0,1]$, the use of estimate (10) does not evidently lead to the similar statement.

In the numerical analysis of the problems of nuclear-nuclear interaction, based on the approximate solution of equations of type (1), the question of the possible reduction in the number of computational points with preservation of admissible accuracy of results for various potential functions $K(x, y)$ is important. We note in this connection that although the convergence of the approximation of equation (1) by the given scheme is proved, nevertheless the differential properties of the initial data do not allow one to state a very high convergence of the process. This and the practice of numerical solution of the Lippman-Schwinger equation show the need of additional reserves of increasing the accuracy of the scheme. Since the Yukawa potential considered here simulates the basic properties of the majority of such potentials, we will try here to analize by this example some computational aspects of the scheme suggested.

From the point of view of the optimization of computational processes, when solving similar problems it is essential to have a possibly more precise agreement of the dynamics of transformation of the given infinite interval with that of the finite interval with the properties of the corresponding integrand, taking into account the peculiar importance of behavior of the mapping function $y=y(t)$ and also of its derivatives in the neighborhood of $0,+\infty$ and the singular point $x_{0}$.

These properties in turn are expressed by the character of distribution of the quadrature nodes in the neighborhoods indicated. Note that according to the estimates given above the expression

$$
\frac{K(x, y(t)) T\left(y(t) ; x_{0}\right) y^{\prime}(t)}{y^{2}(t)-x_{0}^{2}} \quad(y=-1+1 / t),
$$

for any $x>0$, tends to zero for $t \rightarrow 0$. One can observe that the same happens when $t \rightarrow 1$. Since, according to the known asymptotic properties of the zeros of Jacobi's polynomials, the knots of the Gaussian quadrature are condensed more intensively near the ends of the given 
segment and the coefficients corresponding to them tend to zero faster ( $[10 ; \S \S 8.9,15.3])$, the values of the indicated expression in the quadrature sum will be small in absolute value. This usually promotes the reduction of the accuracy of the scheme for the given number of knots. In this connection the possibility of this scheme of mapping the infinity into the midpoint of $[-1,+1]$ (which essentially was used above in the proof of convergence of the quadrature process) may prove to be its certain computational advantage. Note, however, that when replacing $y(t)=-1+1 / t$ by $-1+1 / t^{1-\varepsilon}$ for the arbitrary small $\varepsilon>0$ the proof of the convergence of the quadrature process does not seem to be evidently possible. ${ }^{5}$ In this sense the rate of approximation of the nodes to the infinity defined by this scheme seems rather acceptable.

As for the similar inconvenience for $t \rightarrow 1$, in this connection the reason of the consideration of the mapping function in the form $y=c(-1+1 / t)$ with some constant $c>1$ (generally dependent on $n$ ) arises. However, since the introduction of such a factor causes the shift of the whole system of knots, its improper choice may make a negative influence on the accuracy of the scheme. Here also the analysis of the asymptotic behavior of the Gaussian knots and the quadrature coefficients with the asymptotics of the expression $K(x, y) T(y)$ near the point $y=0$ gives a certain approximate idea of the rate of the value of $c$ more or less acceptable to the scheme.

Some improvements for the specific values of $c$ may be based on the use of the immediate table values of the quadrature knots and coefficients (see, e.g., $[4,5]$ ). These reasonings are partially heuristic, nevertheless, with the indicated estimates they give rather good approximation to the reality and show that the corresponding tentative constants are approximately 8-10 already for relatively small values of $n$.

On the other hand it is evident that while increasing $c$, the modulus of the derivatives $y^{\prime}(t), \quad y^{\prime \prime}(t)$ increases over the entire given segment (near the singular point $x_{0}$ among them), which can promote the increase of the modulus maximum of the derivative of the corresponding integrand and via that decrease of the accuracy of the scheme generally for all possible potentials (this is reflected also in numerical calculations of some test problems).

Starting from those concepts, it seems reasonable for relatively small $c$ to add to the mapping function some terms bounded at infinity, which make the needed shift of only those knots which are near the point corresponding to $t=1$. In the given case, being oriented mainly to the schemes with relatively small number of knots, on this basis we shall consider

$$
y=2\left(-1+1 / t+e-e^{t}\right)
$$

as a mapping function (where both the main part of the map $-1+1 / t$ and the additional term of the local shift $e-e^{t}$ are taken with factor $c=2$.). In calculations we must look to it that for the given $c$ the knots $\in(o,+\infty)$ should not coincide with the considered values of the parameter $x_{0}$.

Below some test examples connected with the computation of the so-called phase of nucleon-nucleon dispersion are given (which in our notations correspond to the expression $\left.T\left(x_{0} ; x_{0}\right) / x_{0}\right)$. Generally the values $-\arctan T\left(x_{0} ; x_{0}\right) / x_{0}$ are calculated by the laboratory system of reference of potential energy $-E$ (see, e.g., [2]), $x_{0}=\sqrt{E / 82.94}$.

In the tables below the corresponding values are given for two known potentials: the Yukawa potential and the so-called phenomenological potential of Reid (see , e.g., [1; p. 229]) for some values of $E$ in the range $1 \leqslant E \leqslant 500$ for different numbers of knots $-N$.

\footnotetext{
${ }^{5}$ Here the reduction of the accuracy of results is observed in the numerical experiment carried out for some values of $\varepsilon$.
} 
Table 1. Yukawa potential

\begin{tabular}{|c|c|c|c|c|c|c|}
\hline$E \backslash N$ & 6 & 8 & 10 & 12 & 16 & 24 \\
\hline 1 & 1.531334 & 1.533273 & 1.530948 & 1.530586 & 1.530414 & 1.530278 \\
40 & .926652 & .919544 & .918908 & .918800 & .918641 & .918563 \\
100 & .751998 & .755777 & .754936 & .754392 & .754267 & .754184 \\
180 & .660076 & .655056 & .655186 & .655309 & .655170 & .655075 \\
400 & .533588 & .532045 & .531624 & .531478 & .531361 & .531279 \\
500 & .494964 & .497845 & .499072 & .499406 & .499375 & .499251 \\
\hline
\end{tabular}

For comparison, the results of the similar calculations are given in Tables 2-3 for the case of Reid potential calculated by both the scheme given here and the scheme ${ }^{6}$ given in [2] for corresponding values of $E$ (remember that in Table 2, like in Table 1 , we mean $N=n / 2$ ):

Table 2. Reid potential (using given scheme)

\begin{tabular}{|c|c|c|c|c|c|c|}
\hline$E \backslash N$ & 6 & 8 & 10 & 12 & 16 & 24 \\
\hline 24 & .849071 & .858220 & .863522 & .861213 & .860602 & .860637 \\
48 & .675120 & .682732 & .686985 & .685110 & .684689 & .684604 \\
96 & .429926 & .438677 & .442377 & .440622 & .440159 & .440193 \\
144 & .252024 & .261073 & .265246 & .263467 & .262989 & .263032 \\
208 & .069247 & .077849 & .082525 & .080779 & .080274 & .080326 \\
352 & -.227001 & -.218997 & -.213623 & -.215786 & -.216432 & -.216381 \\
\hline
\end{tabular}

Table 3. Reid potential (table from [2])

\begin{tabular}{|c|c|c|}
\hline$E \backslash N$ & 16 & 24 \\
\hline 24 & .86182 & .86071 \\
48 & .68560 & .68466 \\
96 & .44111 & .44080 \\
144 & .26395 & .26310 \\
208 & .08135 & .08040 \\
352 & -.21511 & -.21630 \\
\hline
\end{tabular}

According to Tables 1 and 2, the results are stabilized sufficiently rapidly beginning from relatively small $N$. The comparison of Tables 2 and 3 coordinates with the concept that the scheme suggested in this paper is tentatively more economical.

Proceeding from certain concepts, a further improvement of the scheme is possible by choosing the mapping function depending on $n$ and the values of the parameter $x_{0}$. The establishment of such dependence is, however, rather difficult and may represent a subject of separate investigation.

\footnotetext{
${ }^{6}$ In [2] the results of computations are given only for $N=16$ and $N=24$.
} 


\section{References}

[1] J. E. Brown and E. D. Jackson, The Nucleon-Nucleon Interaction, Atomizdat, Moscow, 1979, in Russian.

[2] M. I. Hartel and F. Tabakin, Nuclear saturation and smoothness of nucleon-nucleon potentials, in: Nuclear Physics, A158, North Holland, Amsterdam, 1970, pp. 1-42.

[3] M. Holdberger and K. Watson, The Theory of Collision, Mir, Moscow, 1967, in Russian.

[4] A. C. Kronrod, The Knots and Weights of Quadrature Formulas, Nauka, Moscow, 1954, in Russian.

[5] V. I. Krylov and L. Shulgina, A Reference Book in Numerical Integration, Nauka, Moscow, 1966, in Russian.

[6] V. I. Lebedev and O. V. Baburin, On a calculation of the integrals in principal value sense, weights and knots of gauss quadrature formulas, Zh. Vychisl. Mat. Mat. Fiz., 5 (1965), No. 3, pp. 454-462, in Russian.

[7] R. Newton, The Theory of Dispersion of Waves and Particles, Mir, Moscow, 1969, in Russian.

[8] J. G. Sanikidze, On quadrature formulas for singular integrals, Proc. of the Computer Center of the Academy of Sciences Of GSSR, 11 (1972), No. 1, pp. 110-120, in Russian.

[9] E. Schmid and K. Tsigelman, The Problem of Three Solids in Quantum Mechanics, Nauka, Moscow, 1979, in Russian.

[10] G. Szegö, Orthogonal Polynomials, Fizmatgiz, Moscow, 1959, in Russian.

[11] J. Taylor, The Theory of Dispersion, Mir, Moscow, 1975, in Russian.

Received 22 Aug. 2000 